\title{
SNPs Genotyping Technologies and Their Applications in Farm Animals Breeding Programs: Review
}

\author{
Hamed Kharrati Koopaee $^{1 *}$ and Ali Esmailizadeh Koshkoiyeh ${ }^{2}$ \\ ${ }^{1}$ Institute of Biotechnology; Shiraz University - Iran. ${ }^{2}$ Department of Animal Science; Shahid Bahonar University of \\ Kerman; Kerman - Iran
}

\begin{abstract}
Single nucleotide polymorphisms (SNPs) are DNA sequence variations that occur when a single nucleotide: adenine $(A)$, thymine $(T)$, cytosine $(C)$ or guanine $(G)$ in the genome sequence is altered. Traditional and high throughput methods are two main strategies for SNPs genotyping. The SNPs genotyping technologies provide powerful resources for animal breeding programs. Genomic selection using SNPs is a new tool for choosing the best breeding animals. In addition, the high density maps using SNPs can provide useful genetic tools to study quantitative traits genetic variations. There are many sources of SNPs and exhaustive numbers of methods of SNP detection to be considered. For many traits in farm animals, the rate of genetic improvement can be nearly doubled when SNPs information is used compared to the current methods of genetic evaluation. The goal of this review is to characterize the SNPs genotyping methods and their applications in farm animals breeding.
\end{abstract}

Key words: SNPs genotyping, Traditional and high throughput methods, Animal breeding

\section{INTRODUCTION}

DNA markers have a main potential role in animal breeding programs. The use of DNA markers has a revolutionary impact on gene mapping and generally, on the genetics of all the animal and plant (Dodgson et al. 1997). In the past decades, advances in molecular genetics have led to the development of DNA marker applications on the plant and animal. For example, the identification of multiple genes or genetic markers associated with the genes, which affect the traits of interest in livestock, including the genes for single-gene traits and QTL or genomic regions that affect quantitative traits. This has provided opportunities to enhance the response to selection (Dekkers 2004).

DNA markers are categorized into two main groups; i) PCR based markers such as microsatellites and SNPs, ii) Non-PCR based markers. In the later group, the restricted enzymes have main role for their amplification and production for example, restricted fragment length polymorphism, or RFLP markers (Avise 2004). Single nucleotide polymorphisms (SNPs) involve the substitution of one nucleotide for another. In the other words, SNP marker is just a single base change in a DNA sequence (Beuzen et al. 2000). SNPs include more than $90 \%$ of all differences between the individuals; therefore, they are the best genetic variation resource for population studies and genome mapping (Frohlich et al. 2004). Genomic selection using the SNP markers is a powerful new tool for genetic selection (Seidel 2010). Advantages of the SNP markers include: 1 . most of the SNP markers are located in coding area of DNA; therefore, they affect protein function directly, 2. SNPs are more suitable than

\footnotetext{
*Author for correspondence: h.kharrati.ko@gmail.com
} 
microsatellites for high throughput genetic analysis, 3. They are stably inherited than other DNA markers, making them more suited as long term selection markers, and 4 . they are prevalent and provide more potential markers near the locus of interest than other types of polymorphism. The problem of the SNPs is the biallelic nature of these markers, which means that there are usually only two alleles in a population. Consequently, the information content per SNP marker is lower than multiallelic markers such as microsatellite markers. Five SNP markers provide similar information to one microsatellite marker (Beuzen et al. 2000).

SNPs genotyping is performed using two main methods, the traditional and high throughput methods. The traditional gel-based approach uses standard molecular techniques, such as amplification refractory mutation system (ARMS), restriction digests and various forms of gel electrophoresis (e.g., RFLP), denaturing gradient gel electrophoresis (DGGE) and singlestrand conformation polymorphism (SSCP). High throughput methods include allele discriminationmethods (Allele-Specific Hybridization, Allele-Specific Single-BasePrimer Extension), High-throughput assay chemistry (Flap endonuclease discrimination, Oligonucleotide ligation), DNA arrays, pyrosequencing and light cycler. In this paper, these methods are reviewed, followed by discussing on the applications of SNPs technology in farm animals breeding programs.

\section{TRADITIONAL GEL-BASED APPROACH}

\section{Amplication Refractory Mutation System (ARMS)}

The ARMS technique is used for the recognition of unknown mutations. The ARMS method relies on allele specific PCR. Two different forward primers are designed with differing 39 nucleotides to complement the two expected alleles. Synthesis occurs during the PCR only if correct base pairing is present throughout the whole primer site. With proper primer design, the two different SNPspecific primers can be made to produce the distinguishable products, possibly using different 59 fluorescent labels, or different 59 extensions to produce the product length differences. Multiplexing, using multiple sets of SNP-specific primers to give a range of identifiable products, perhaps by size and colour fluorescence, can lead to efficient screening of sufficient numbers (Dearlove 2002). Shahid Zadehet al. (2011) showed the application of ARMS in the identification of unknown mutations. In this study, 138 Persian Arab horses were tested by ARMSPCR. DNA-PK is the catalytic subunit of a nuclear DNA-dependent serine/threonine protein kinase, called DNA-PK and has a critical role in terms of antigen recognition. It is absolutely required for the development and survival of B and T cells. It is known that the deletion of a five-base pair TCTCA results in a mutation in frame-shift at codon 3155 and premature stop codon. Foals that are homozygous for mutation allele are affected by the severe combined immunodeficiency (SCID). A foals affected by the SCID appears to be healthy at birth, but during the first month of birth, the symptoms appear and due to the opportunistic infections, dies after five months. Since the disease inherits as autosomal recessive, transmission of defect allele could be prevented by carrier recognition leading to a proper management in reproductive program. Based on the results of Shahid Zadeh et al. (2011) one of the foals studied was recognized as affected and the others as unaffected (Fig. 1).

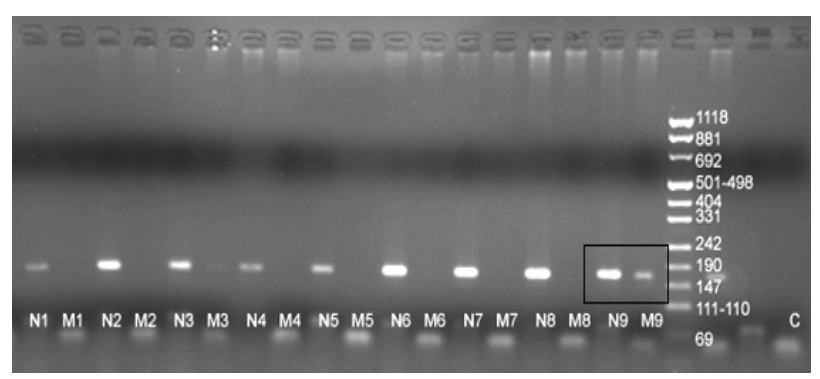

Figure 1 - Individual 9 was recognized as affected (Shahid zadeh et al. 2011).

$\mathrm{N}$ : ARMS- PCR product using of normal primes; M: ARMS- PCR product using of mutation primes; C: Negative control; Ladder: PUC MIX 8.

\section{Restericted Fragment Length Polymorphism (RFLP)}

RFLP technique is used for the known mutations and SNPs. This approach consists of two main steps. First, the target DNA involving the SNP is amplified using the standard PCR. Second, the PCR product is digested using restriction enzymes. Nucleotide changes occur in all the eukaryotic genomes. If the change results in the creation, or abolition of a restriction endonuclease recognition 
site, then the DNA sequence acquires, or loses the ability to be cleaved by a particular restriction endonuclease. If a recognition site is absent, then the digestion with the relevant restriction enzyme generates a long fragment. If a recognition site is present, then the digestion with the relevant restriction enzyme generates two shorter fragments. If the recognition site is in only one of two parental alleles, digestion will produce two different electrophoretic patterns: a long fragment and two shorter fragments (Beuzen et al. 2000). The most undesirable features of this approach include 1. most mutations do not result in the abolition, or creation of restriction endonuclease sites, making such mutations impossible to detect by RFLP analysis, 2 . in some cases, the digestion step requires a long time and high temperature (Avise 2004). So far, many SNPs have been detected using RFLP, e.g. DGAT1, a major gene in dairy cow herds. DGAT1 gene codes diacylglycerol-acyltransferase enzyme, which plays a main role in the triglyceride synthesis and subsequently milk composition and production. A transition mutation (SNP) in this gene results in the substitution of guanine by adenine in the gene leading to the substitution of lysine by alanine in diacylglycerol-acyltransferase enzyme (Grisart et al. 2002). Kharrati et al. (2011) detected three genotypes of this gene using RFLP technique in Iranian Holstein cattle population. The DNA restriction fragments obtained for DGAT1 gene using the $C f r I$ enzyme were 411 bp (no digestion) for the KK genotype, 203 and 208 bp for the AA, 411 and 203 or 208 bp for the KA genotype (Fig. 2) (Kharrati et al. 2011).

\section{Denaturing Gradient Gel Electrophoresis (DGGE)}

DGGE is based on the melting point of double stranded DNA that is influenced by the presence of a mismatch. When the melting point is reached in a poly-acrylamide gel containing a gradient of denaturant, the electrophoretic mobility is reduced. In some versions of this technique, denaturing high performance liquid chromatography (DHPLC) is used for the separation of the hetero duplex and homo duplex strands (Liu et al. 1998). Diez et al. (2001) used the DGGE to study the diversity of marine Pico eukaryotic. In this study, two eukaryote-specific primer sets targeting different regions of the 18S rRNA gene were tested and DGGE was performed with a DGGE2000 system (CBS Scientific Company).

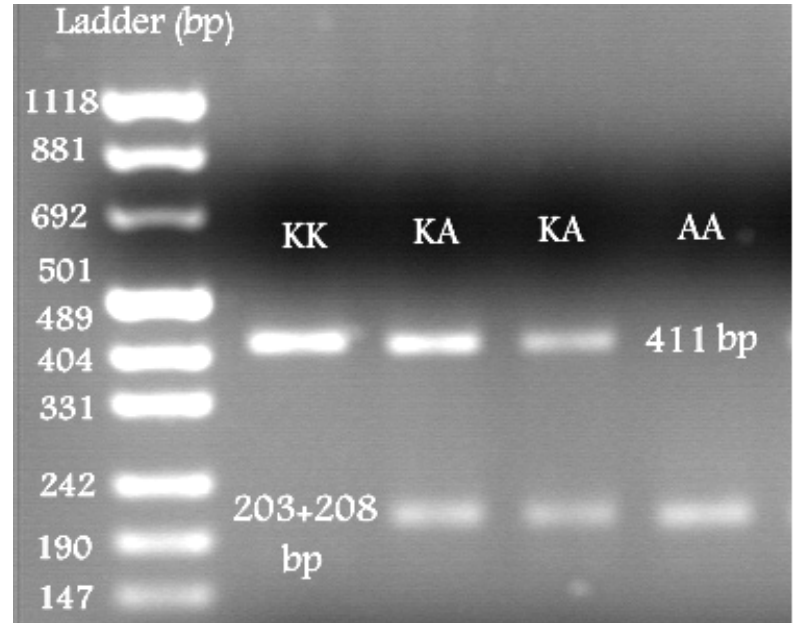

Figure 2 - DGAT1 genotyping in Iranian Holstein cattle population.

Ladder: PUC MIX 8 (Kharrati et al. 2011).

\section{Single-Strand Conformation Polymorphism (SSCP)}

SSCP technique is performed for the unknown SNPs and mutations in DNA molecule. The SSCP is based on the changes in secondary structure(s) in single-stranded DNA fragments caused by a change in sequence, which are detected as alterations in the fragment mobility by gel electrophoresis. This method is based on the differences in nucleotides. It is one of the easiest screening procedures to perform, is very cost effective and has a reportedly high mutation detection rate (70-95\% for some genes). However, SSCP is intensive and analytical steps are performed before and during the electrophoresis, the concentrations of DNA template and primers, running temperature and time may affect the detection of mutations and reproducibility (Shojaei et al. 2010). In this technique, primers are designed to produce the fragments up to $300 \mathrm{bp}$ in length. These products are heat denatured and snap cooled, and the resulting single stranded products are loaded on a non-denaturing acrylamide gel. Products may be radio-labeled, fluorescently labeled, or if unlabeled, they could be detected by silver staining. The differing conformations brought about by the mutation lead to a different DNA mobility due to the intra-strand secondary structure, so the banding patterns are visualized and compared with the controls of known variants (Orita et al. 1989). Shojaei et al. (2010) investigated the association of growth trait and leptin gene polymorphism in kermani sheep using the SSCP technique. In this study, 275 bp region 
of exon3 of leptin gene was amplified using the specific primers, then the PCR products were resolved by SSCP. For SSCP analysis, 7.0 $\mu \mathrm{L}$ aliquot of each amplicon was mixed with $7.0 \mu \mathrm{L}$ of loading dye $(98 \%$ formamide, $10 \mathrm{mM}$ EDTA, $0.025 \%$ bromophenol blue, and $0.025 \%$ xylenecyanol). After denaturation at $95^{\circ} \mathrm{C}$ for $5 \mathrm{~min}$, samples were rapidly cooled on wet ice for $10 \mathrm{~min}$ to prevent the reannealing of the single-stranded product and then loaded on $8 \%$ acrylamide. The results of this study indicated significant effect of leptin gene on the growth traits.

\section{HIGH THROUGHPUT METHODS}

Many high throughput methods are available for SNPs genotyping that offer a unique combination of scale, accuracy, throughput, and cost (Gabriel et al. 2009). However, the goal in this section is to introduce the important methods.

\section{ALLELE DISCRIMINATION METHODS}

\section{Allele-Specific Hybridization (TaqMan ASSAY)}

The TaqMan assay uses the intrinsic 5 ' nuclease activity of Taq DNA polymerase to generate a fluorescent signal from a short allele specific oligonucleotide (ASO) probe. Two ASO probes are required, one specific for each allele. Each probe contains a unique donor fluorophore and a common acceptor fluorophore, and is short enough for the donor to be quenched when the probe is intact (either hybridized, or in solution). However, when the probe hybridizes to the PCR template, the 5'exonuclease activity of Taq DNA polymerase digests it, thus releasing the two fluorophores into solution and eliminating the quenching effect (Twyman 2005). This assay is the 59 nuclease assay. The allelic discrimination is based on the characteristic 59 to 39 exonuclease activity of Taq DNA polymerase (Holland et al. 1991; Livak et al. 1995). PCR is performed using the flanking primers, including the fluorescent oligonucleotide probes in a homogeneous assay. The probes consist of a 59 reporter dye and a 39 quencher dye, and are specific to the region containing the base change in the region to be amplified. The 59 nuclease activity cleaves the probe if hybridization occurs, releasing the reporter from the quencher. Two different probes with different fluorogenic reporters are put in the reaction for allele discrimination, one specific to complement each of the variant alleles to be typed. If there is a mismatch between the probe and target DNA sequence, the hybridizationis significantly reduces, therefore stops the cleavage of reporter from quencher and releases the fluorescent signal.

\section{Allele-Specific Single-Base Primer Extension}

Primer extension is a very robust allelic discrimination mechanism. It is highly flexible and requires the smallest number of primers or probes. Several stages are required for any protocols designed around the principle of primer extension. Products covering the SNP region are amplified by the PCR, and any remaining amplification primers and dinucleotide triphosphates (dNTPs) are inactivated, or removed prior to minisequencing (Syvanen 1999). Primer extension, also known as mini-sequencing, forms the basis of a number of methods for allelic discrimination. An extension primer is annealed $5 \mathrm{k}$ to a SNP, either adjacent to but not including the SNP, or several bases upstream of the SNP. The primer is then extended for one, or several nucleotides to include the SNP site. In the case of single base extension (SBE), a primer is annealed adjacent to a SNP and extended to incorporate a dideoxynucleotide (ddNTP) at the polymorphic site (Jenkins and Gibsone 2002). If dideoxynucleotide triphosphates labeled with different dyes are used, single base extension (SBE) products can be run on a fluorescent sequencer, either gel or capillary based, for electrophoresis, where data can be visualized and collected for the analysis (Dearlove 2002).

\section{HIGH-THROUGHPUT ASSAY CHEMISTRY}

\section{Flap Probe Cleavage Approach}

A new molecular mechanism for single base detection has been recently described. This approach is based on the observation that flap endonucleases (also called cleavages) isolated from the archaic recognize and cleave a structure formed when two overlapping oligonucleotides hybridize to a complementary DNA target. When the downstream oligonucleotide is designed with a 'flap' consisting of non-complementary sequence, this stretch of DNA is cleaved in the presence of an upstream 'invader' oligonucleotide and target DNA. If the cleaved 'flap' is used as the 'invader' oligonucleotide in a secondary assay with a second 'flap' probe and a synthetic template, one can amplify the signal without amplifying the genomic 
DNA target. The final detection can be based on the fluorescence intensity changes when the fluorescence resonance energy transfer (FRET) probe is cleaved (Lyamichev et al. 1999). The main advantages of this approach are the possibility of eliminating PCR amplification, the low cost of unlabelled allele-specific probes and the relative simplicity of the reaction protocol. The only drawback is the need for relatively large amounts of genomic DNA in the invader assay when PCR is not used.

\section{Oligonucleotide Ligation Assay (OLA)}

The OLA assay was described for the first time by Landegren et al. (1988). This assay is performed by designing two oligonucleotides specific for each allele (Tobeet et al. 1996). In the other words, OLA involves ligation of two oligonucleotides, hybridized to a DNA template, one of which is allele specific such that it will only form part of a ligated product if it is complimentary to the target sequence (Jenkins and Gibson 2002). For the oligonucleotide ligation assay, two primers are designed that are directly next to each other when hybridized to the complementary target DNA sequence in question. The two adjacent primers must be directly next to each other with no interval, or mismatch, for them to be covalently joined by ligation (Dearlove 2002).

\section{DNA Array}

DNA chips and microarrays, of immobilized oligonucleotides of known sequences, which differ at specific sites of individual nucleotides (at the site of SNP), can also be used for the detection of SNPs. The technique is actually suitable to score several SNPs in parallel from each sample in a multiplexed fashion. It makes use of the technique of sequencing by hybridization (SBH) and involves tiling strategy. Four oligonucleotides in a column of an array differ only at the SNP site and only one would be fully homologous. When such an array is hybridized with the PCR product, the perfect match allows the binding and mismatched products would be washed away. The perfect match in each case can be detected through a detection system (Gupta et al. 2001).

\section{Pyrosequencing}

Pyrosequencing is particularly suitable for SNP genotyping, since genotyping of previously identified SNPs by this method requires sequencing of only a few nucleotides (1-5 bp)
(Gupta et al. 2001). Pyrosequencing technology presents SNPs in the context of the surrounding sequence. This guarantees that each individual analysis is correct, without the need for other controls. In a clinical context, this level of certainty is highly valuable, especially as the genetic methods developed in today's research mature into tomorrow's genetic tests. Pyrosequencing is a recent rapid re-sequencing technology, in which template-mediated, oligonucleotide primed incorporation of nucleotides by a polymerase, is monitored by a measure of pyrophosphate (PPi) release. The four possible nucleotides are injected sequentially in the reaction mixture and the succession of successful incorporations, recorded on a program, gives the sequence (Ronaghi 2001). Comparison of the sequences with a reference enables to score SNPs. An advantage of the method is that any new polymorphism can be detected. However, special equipment is needed for the injection of the nucleotides (Vignal et al. 2002).

\section{SNP Detection/Genotyping Using the Light Cycler System}

The Light-Cycler Instrument enables both the amplification and the real-time on-line detection of a PCR product, thus allowing accurate quantification. The system also provides a unique and innovative approach perfectly suited for the detection and genotyping of single nucleotide polymorphisms: the melting curve analysis feature. During the melting curve analysis, the Light Cycler Instrument monitors the temperaturedependent hybridization of the sequencespecific hybridization probes to single stranded DNA (Alderborn 2000). During the PCR, a DNA fragment of the respective gene is amplified with specific primers fromanimal genomic DNA. The ampliconis are detected by the fluorescence using specific pairs of hybridization probes. Hybridization probes consist of two different oligonucleotides that hybridize to an internal sequence of the amplified fragment during the annealing phase of PCR cycles. One probe is labeled at the $5^{\prime}$-end with a Light Cycler-Red fluorophore (Light Cycler-Red 640 or Light Cycler-Red 705) and, to avoid extension, is modified at the $3^{\prime}$-end by phosphorylation. The other probe is labeled at the 3'-end with fluorescein. Only after hybridization to the template DNA, the two probes come in close proximity, resulting in fluorescence 
resonanceenergy transfer (FRET) between the two fluorophores. During the FRET, fluorescein, the donor fluorophore, is excited by the light source of the Light Cycler Instrument and part of the excitation energy is transferred to Light CyclerRed, the acceptor fluorophore. The emitted fluorescence of the Light Cycler-Red fluorophore is measured (Suzanne 2000).

\section{APPLICATION OF HIGH THROUGHPUT SNPS GENOTYPING METHODS IN FARM ANIMALS BREEDING}

Advances of molecular knowledge about the genes and their biology function refer to several past decades. Therefore, breeders have enhanced response to selection for the traits by selecting the individuals according to records and pedigree information. Breeders have tried to enhanced "breeding values" for economic traits. The traditional breeding programs were obtained as substantial results for the genetic trends. For example, in Holstein dairy cattle, milk production still increases by $110 \mathrm{~kg}$ per animal per year. In pig production, the kilograms of feed required to produce a kilogram of pork, known as feed conversion, is estimated to have decreased by $50 \%$ between the 1960s and 2005. Although these results illustrate powerful examples that can be achieved through the traditional breeding methods, but there are several problems with the traditional methods. For instance, many economic traits are difficult to measure (e.g., somatic cell score and feed efficiency); some traits have a low heritability, or cannot be quickly recorded (e.g., disease resistance and fertility), thereby traditional methods are successful but limited (Eggen 2012). The rapid improvements in high-throughput methods for SNPs genotyping technologies and the development of the SNP arrays accompanied by reduced costs for genotyping and for sequencing have opened the possibility of using genomic information in livestock breeding programs through the novel method for genetic selection called genomic selection.

Economic traits in farm animals are polygenic traits that are controlled by many loci, each with different effect. Genomic selection refers to selection decisions based on the genomic estimated breeding values (GEBVs) (Meuwissen et al. 2001). The GEBVs are calculated as the sum of the effects of dense genetic markers that are approximately equally spaced across the entire genome, thereby potentially capturing most of the quantitative trait loci that contribute to variation in a trait (Pryce et al. 2012). Genomic enabled selection is a method of marker-assisted selection (MAS) based on linkage disequilibrium (LD) between the markers and quantitative traits loci (QTL). Heritability, LD and number of animals have main role in genomic selection. Generally, the success of genomic selection depends on these factors (Brito et al. 2011). Genomic selection estimates a prediction equation in a reference population with genotype and phenotype data. This prediction equation can then be used to predict the breeding values in the animals without phenotype data. In fact, the use of genomic selection can lead to calculate accurate EBV before sexual maturity. This means that breeders can identify superior animals at earlier age (Schefers et al. 2012). The revolution in genotyping provided by the high-density SNP and the associated reduction in the cost have resulted in large numbers of individuals with genome-wide genotypic data. Thus, genomic selection can be widely implemented in farm animals once the accuracy of genomic selection is high enough (Goddard 2012).

There are many studies about the genomic selection in farm animals. For instance, Schefers et al. (2012) discussed the possible applications of this technology in dairy industry and concluded a greater accuracy of predicted genetic merit for young animals, a shorter generation interval because of heavier use of young, genetically superior males and females, and an increased intensity of selection because breeder could use genomic testing to screen a large group of the potentially elite animals. By increasing the accuracy and intensity of the selection and shortening the generation interval, the rate of genetic progress for economically important dairy traits can be approximately doubled (Schefers et al. 2012). Preisinger (2012) made a study on poultry and showed that the genomic selection provided precise tools, which could be used in growing animals without performance testing, which increased the speed and accuracy of selection decisions. Genomics helped to determine which of the day-old chicks had the highest chance of transmitting superior genetics for all the traits of economic importance to meet the next generation of pure lines, or grandparents. 
The other application of high throughput methods and SNPs genotyping is the identification of the genes responsible for disease resistance in poultry. For example, Cheng et al. (2012) performed a study on the Marek's disease. The Marek's disease is one of the most important diseases in poultry industry. In this study, the analysis of genomic sequences from two experimental lines divergently selected for the Marek's disease genetic resistance allowed inference about the region under the selection as well as potential causative polymorphism. These new combined approaches have resulted in a large number of high-confidence genes conferring the Marek's disease resistance reflecting the multi genic nature of this trait, which expands the biological knowledge and provides corresponding SNPs that can be directly evaluated for their genetic contribution towards disease resistance. Dalloul (2010) reported 600000 SNPs published in turkey genome poultry. More recently, they have reported the identification of 5.49 millions SNPs by evaluating 11 lines of turkeys including seven lines from the commercial breeders.

There are a number of whole-genome single nucleotide polymorphism (SNP) chips developed for important agricultural species. For example, Illumina Inc provides public and commercial SNP chips for cattle (BovineSNP50v2; 54609 SNPs), sheep (OvineSNP50; 52241 SNPs), chicken (57636 SNPs) and pig (PorcineSNP60; 62163) (Eggen, 2012). Generally, the success in the use of highly dense commercial DNA markers (SNP chips) depends on several factors, which include (i) the chips need to be developed because many composite synthetic commercial breeds are available; also, some breeds are combination of multibreeds, (ii) many commercial species are genetically separated and their environments are not similar; relevant differences are expected in the effects of DNA markers in separated populations (this would be an expression of genotype $\times$ environment interactions), and (iii) the cost of genotyping is a major problem in the genomic technology. Selections for the traits are very different. For example, the selection for milk is very different from the selection for beef, and the value of selected replacement of beef bulls, which are normally used in natural mating in more than $90 \%$ of the herds, is very different from that of dairy bulls used in artificial insemination. Hence, genotyping for beef bulls and heifers has not been affordable with the technology prices in
2011 (Hugo et al. 2012). Goddard (2012) discussed the uses of genomics in livestock agriculture and reported the strong need to increase the biological efficiency of livestock production to meet the rising costs of inputs expected in the future. Long-term genetic improvement, using genomics and reproductive technologies, could achieve part of the increased efficiency needed.

Several projects of genomic selection and genome wide association have been completed in commercial domestic animals. For example, Genome-wide association studies in dairy cattle have been completed for several traits and types of traits in different regions of the world. Australia has taken the lead in identifying the genomic regions associated with milk production (Bolormaa et al. 2010; Pryce et al. 2010). Several studies have also been completed in the United States and Canada (Wiggans et al. 2009; Cole et al. 2011) and China (Jiang et al. 2010). Recently, Fulton (2012) has discussed several challenges in the application of genomic information. For example, the cost of genotyping is a main challenge in genomic selection. Although the costs of genotyping each SNP are declining rapidly, the cost per sample is not. The costs of the $42 \mathrm{~K}$ and $60 \mathrm{~K}$ chicken SNP were US\$200 per sample. Although the upcoming 600K SNP chip may have 10 times the number of SNP, the cost per sample is expected to be approximately US\$250. Initial genomic selection requires large training panels involving 5,000 animals or more, resulting in a cost of US\$ 1.25 million for the training panel alone. Lack of basic research is one of the challenges. It is very important to understand exactly how variation is influencing a trait, but it is important to understand how the animal is affected in a systems biology context. Research in the "genomic revolution" is expensive, and while it gets funded because it is novel, or revolutionary, the basic sciences are being neglected. One of the biggest challenges for the application of genomic information is integrating this information into existing breeding programs. Extensive pedigree records, along with trait information and predicted breeding values are currently used in selection programs. Genomic information is currently scarce and still relatively expensive. The key to successful implementation depends on the ability of the breeding organization to combine these sources of information in an optimal way to maximize the accuracy of prediction or the 
breeding value. Finally, loss of genetic variation is other challenge in this technology. Genetic variation is the key to understanding how DNA changes can influence the traits. Fulton and Delany (2003) summarized the poultry genetic losses that had occurred over the preceding 15 years. Further erosion has occurred since with multiple research institutions eliminating all poultry genetic stocks.

As mentioned above, the primary goal of wholegenome selection is to enhance the genetic progress through more accurate selection of the individuals for breeding and subsequent improvement of the next generations. Possible additional applications include the parentage identification, decreasing the number of animals selected for breeding, decreasing the number of progeny to be phenotyped, and decreasing the generation interval because selection candidates could be identified at an earlier age. Each of these applications has the potential to radically change the breeding structures of elite populations (Fulton 2012).

\section{CONCLUSION}

Traditional SNPs genotyping technologies are suitable for the study of few SNPs in the genome. Use of these techniques could help to study the association between the special SNPs and economic traits. High throughput methods provide high density of SNPs. By the identification of SNPs, exploitation of novel genomic-enabled selection methodologies in farm animals could be possible. Genomic selection can lead to the decreased generation interval and increased selection intensity, thus designing a cost-effective breeding program.

\section{REFERENCES}

Alderborn A, Kristofferson A, Hammerling U. Determination of single nucleotide polymorphisms by real-time pyrophosphate DNA sequencing. Genome Res. 2000; 10: 1249-1258.

Avise JC. Molecular markers natural history and evolution. 2nd. Georgia: University of Georgia. 2004.

Eggen A. The development and application of genomic selection as a new breeding paradigm. Anim Frontiers. 2012; 2(1): 10-15.
Beuzen ND, Stear MJ, Chang KC. Molecular markers and their use in animal breeding. Ver J. 2000; 160: 42-52.

Brito FV, Neto JB, Sargolzaei M, Cobuci JA, Schenkel FS. Accuracy of genomic selection in simulated populations mimicking the extent of linkage disequilibrium in beef cattle. BMC Genetics. 2011; 12(80): 1-10.

Bolormaa S, Pryce JE, Hayes BJ, Goddard ME. Multivariate analysis of a genome-wide association study in dairy cattle. J Dairy Sci. 2010; 93: 3818-3833.

Cheng HH, Mac-Eachern CS, Subramaniam S, Muir MW. Chicks and single-nucleotide polymorphisms: an entree into identifying genes conferring disease resistance in chicken. Anim Prod Sci. 2012; 52:151-156.

Cole JB, Wiggans GR, Ma L, Sonstegard TS, Lawlor JT, Cooker BA, et al. Genome wide association analysis of thirty-one production, health, reproduction and body conformation traits in contemporary U.S. Holstein cows. BMC Genomics. 2011; 12(408): 1-17.

Dalloul RA, Long JA, Zimin AV, Aslam L, Beal K, et al. Multi-platform next-generation sequencing of the domestic turkey (Meleagris gallopavo): Genome assembly and analysis. Plos Biol. 2010; 8(9): 1-21.

Dearlove AM. High throughput genotyping technologies. Brif Funct Genomics Proteomics. 2002; 1(2): 139-150.

Dekkers JCM. Commercial application of marker- and gene-assisted selection in livestock: Strategies and lessons. J Anim Sci. 2004; 82(13): 313-328.

Diez B, S-Alio C, Terence L, Marsh TL, Massana R. Application of denaturing gradient gel electrophoresis (DGGE) to study the diversity of marine picoeukaryotic assemblages and comparison of DGGE with other molecular techniques. Appl Enviro Microbiol. 2001; 67(7): 2942-2951.

Dodgson JB, Cheng HH, Okimoto R. DNA marker technology: A revolution in animal genetics. Poult Sci. 1997; 76:1108-1114.

Frohlich T, Kirschbaum T, Thoenes U, Furrer F, Dietrichveenstra U, Seller M. The light typer instrument: high through put genotyping of single nucleotide polymorphism. Biochemica. 2004; 2: 9-11.

Fulton JE. Genomic selection for poultry breeding. Anim Frontiers. 2012; 2(1): 30-36.

Fulton JE and Delany ME. Poultry genetic resources operation rescue needed. Sci. 2003; 300:1667-1668.

Gabriel S, Ziaugra L, Diana Tabbaa D. SNP genotyping using the sequenom mass ARRAYiPLEX Platform. Curr Protocol Hum Genet. 2009; 2(12): 1-18.

Goddard ME. Uses of genomics in livestock agriculture. Anim Prod Sci. 2012; 52:73-77.

Grisart B, Coppieters W, Farnir F, Karim L, Ford C, Berzi $\mathrm{P}$, et al. Positional candidate cloning of a QTL in dairy cattle: identification of a missense mutation in the bovine DGAT1 gene with major effect on milk yield and composition. Genome Res. 2002; 12: 222-231.

Gupta PK, Roy JK, Prasad M. Single nucleotide polymorphisms: A new paradigm for molecular marker technology and DNA polymorphism detection with emphasis on their use in plants. Curr Sci. 2001; 80(4): 256-265. 
Holland PM, Abramson RD, Watson R, Gelfand DH. Detection of specific polymerase chain reaction product by utilizing the 59-39 exonuclease activity of Thermus aquaticus DNA polymerase. Proc Natl Acad Sci USA. 1991; 88:7276-7280.

Hugo HM, Casas E, Sterman Ferraz JB, Vega-Murillo VE, van Román-Ponce SI. Opportunities and challenges fromthe use of genomic selection for beef cattle breeding in Latin America. Anim Frontiers. 2012; 291:23-29.

Kharrati H, Mohammad Abadi MR, Ansari S, Esmailizadeh A, Tarang AR, Nikbakhti M (2011). Genetic variation of DGAT1 gene and its association with milk production in Iranian holstein cattle population. Iranian J Anim Sci Res. 2011; 3(2): 185-192.

Jenkins S, Gibson N. High-throughput SNP genotyping. Comp Funct Genomics. 2002; 3: 57-66.

Landegren U, Kaiser R, Sanders J, Hood L. A ligase mediated gene detection technique. Sci.1988; 241: 1077 1080 .

Jiang L, Liu J, Sun D, Ma P, Ding X, Yu Y, Zhang Q. Genome wide association studies for milk production traits in Chinese holstein population. Plos One. 2010; 5:e13661.

Livak KJ, Marmaro J, Todd JA. Towards fully automated genome-wide polymorphism screening. Nat Genet.1995; 9: 341-342.

Livak KJ, Flood SJA, Marmaro J, Giusti W, Deetz K. Oligonucleotides with fluorescent dyes at opposite ends provide a quenched probe system useful for detecting PCR product and nucleic acid hybridization. PCR Methods Appl. 1995; 4: 357-362.

Liu W, Smith DI, Rechtzigel KJ, Thibodeau SN, James CD. Denaturing high performance liquid chromatography (DHPLC) used in the detection of germline and somatic mutations. Nucleic Acids Res.1998; 26: 1396-1400.

Lyamichev V, Mast AL, Hall JG. Polymorphism identification and quantitative detection of genomic DNA by invasive cleavage of oligonucleotide probes. Nat Biotechnol. 1999; 17:292-296.

Meuwissen THE, Hayes BJ, Goddard ME. Prediction of total genetic value using genome-wide dense marker maps. Genetic. 2001; 157:1819-1829.

Orita M, Iwahana H, Kanazawa H, Hayashi K, Sekiya T. Detection of polymorphisms of human DNA by gel electrophoresis as single-strand conformation polymorphisms. Proc Natl Acad Sci. 1989; 86: 27662770.
Preisinger R. Genome-wide selection in poultry. Anim Prod Sci. 2012; 52:121-125.

Pryce JE, Bolormaa S, Chamberlain AJ, Bowman PJ, Savin K, Goddard ME, Hayes BJ. A validated genomewide association study in 2 dairy cattle breeds for milk production. J Dairy Sci. 2010; 93: 3331-3345.

Pryce JE, Daetwyler HD. Designing dairy cattle breeding schemes under genomic selection: a review of international research. Anim Prod Sci. 2012; 52:104114.

Ronaghi M. Pyrosequencing sheds light on DNA sequencing. Genome Res. 2001; 11: 3-11.

Seidel GE. Brief introduction to whole-genome selection in cattle using single nucleotide polymorphisms. Report Fert Develop. 2010; 22(1):138-44.

Schefers JM, Eeigel KA. Genomic selection in dairy cattle: Integration of DNA testing into breeding programs. Anim Frontiers. 2012; 1: 4-9.

Shahid zadeh R, Tarang AR, Ajamiyan F, Haeri-Rohani A. Identification of DNA- PKCS mutation in Arabian horses in Iran. Proceeding: of the first national congress of biotechnology. Isfahan University of technology, Isfahan, Iran; 2011.p. 106-110.

Shojaei M, Mohammad abadi MR, Asadi-Fozi M, Dayani $\mathrm{O}$, khezri A, Akhondi M. Association of growth trait and leptin gene polymorphism in Kermani sheep. J Cell Mol Biol. 2010; 2(1): 67-73.

Suzanne J, Neil G. Fast and flexible single nucleotide polymorphism (SNP) detection with the light cycler system. Biochemitry. 2000; 4: 568-576.

Syvanen AC. From gels to chips: minisequencing human mutation. Hum Mutat.1999; 13:1-10.

Tobeet VO, Taylor SL, Nickerson DA. Single-well genotyping of di allelic sequence variations by a twocolor ELISA- based oligonucleotide ligation assay. Nucleic Acids Res. 1996; 24:3728-3732.

Twyman R. Single Nucleotide Polymorphism (SNP) genotyping techniques. Encyclopedia Diagnostic Genomics Proteomics. 2005; 6: 1202-1207.

Vignal A, Milan D, Sancristobal M, Eggen A. A review on SNP and other typesof molecular markers and their use in animal genetics. Genet Sel Evol. 2002; 34: 275-305.

Wiggans GR, Sonstegard TS, VanRaden PM, Matukumalli LK, Schnabel RD, Taylor JF, et al. Selection of singlenucleotide polymorphisms and quality of genotypes used in genomic evaluation of dairy cattle in the United Statesand Canada. J Dairy Sci. 2009; 92: 3431-3436. 